\title{
Evaluación del estado de aplicación del Programa Nacional para la Conservación en Colombia del Oso Andino (Tremarctos ornatus)
}

\author{
Assessing the implementation of the \\ National Conservation Program of the Andean Bear \\ (Tremarctos ornatus) in Colombia
}

\author{
David Rodríguez-Castroํㅜ, Susy Contento ${ }^{1}$, Diego Grajales ${ }^{1}$, Daniel Rodríguez ${ }^{2}$, \\ Shisley Reyes ${ }^{2}$, Nicolás Reyes-Amaya ${ }^{2}$, Claudia Rodríguez ${ }^{3}$
}

\begin{abstract}
Resumen
Los programas de conservación de especies amenazadas constituyen una herramienta útil para la planeación y ejecución de acciones coordinadas que influyan en el mejoramiento de la salud de las poblaciones de estas especies. Sin embargo, en Colombia es desconocido el impacto real que estos programas tienen al interior de las instituciones encargadas de ejecutarlos. Objetivo: Realizar una evaluación del estado de aplicación del Programa Nacional para la Conservación en Colombia del Oso Andino (PNOA) al interior de 26 corporaciones autónomas regionales (CAR) y la Oficina de Planeación de Parques Nacionales Naturales, Unidad Administrativa Especial de Parques Nacionales Naturales (UAESPNN). Metodología: Se realizó una revisión cuantitativa mediante los criterios de semáforo, sobre la información obtenida de 42 documentos institucionales de planeación y de las respuestas al requerimiento 2100-2-91674 de 2009 del Ministerio de Ambiente, Vivienda y Desarrollo Territorial (MAVDT), así como también de entrevistas con funcionarios. Resultados: Con excepción de una, la totalidad de las CARs presentaron información sobre la ejecución del programa en sus jurisdicciones $(96,2 \%)$. La oficina de UAESPNN no presentó información física o virtual de sus jurisdicciones, impidiendo su inclusión integral en el análisis cuantitativo. En total, la línea de acción del PNOA con mayor porcentaje de aplicación directa fue la línea I (conservación in situ: 30,8\%), la de mayor porcentaje de aplicación indirecta fue la línea $\mathrm{V}$ (fortalecimiento institucional: $88,5 \%$ ) y la de mayor porcentaje de no aplicación fue la línea II (conservación ex situ: 42,3\%). Conclusiones: El PNOA presenta una ejecución mayoritariamente indirecta, donde $61,5 \%$ de las CARs evaluadas sólo habían desarrollado actividades indirectas, asociadas con la ejecución de otros programas nacionales de conservación. Se evidencia un vacío administrativo al interior de las CARs en relación con la ejecución del PNOA, en donde las acciones ejecutadas por las mismas en relación con este provienen más de acciones coyunturales que de la planeación institucional, reflejando que este programa no ha sido asimilado aún de forma estructural.
\end{abstract}

Palabras clave: Autoridades ambientales, Oso andino, Programa Nacional de Conservación.

\begin{abstract}
Conservation programs for threatened species constitute a useful tool for the planning and execution of coordinated actions for the improvement of the population status of those species. Nevertheless, in Colombia it is unknown the real impact that those programs represent inside the institutions in charge of its execution. Objective: To conduct an assessment of the state of implementation of the National Conservation Program (PNOA) for the Andean Bear (Tremarctos ornatus) in Colombia into 26 regional autonomous corporations (CAR's) and the Planning Office of National Natural Parks, called Special Administrative Unit of National Natural Parks (UAESPNN). Methodology: A quantitative review was

\footnotetext{
Antropólogo, Fundación para la Investigación, Conservación y Protección del Oso Andino (Fundación Wii). e-mail: lordnagaro@gmail.com,cunuba@gmail.com,dac5002@hotmail.com

2 Biólogo, Fundación para la Investigación, Conservación y Protección del Oso Andino (Fundación Wii). e-mail: danielosito85@hotmail.com, shisdre@hotmail.com,nicolas.reyes2@gmail.com

3 Biólogo, Ministerio de Ambiente y Desarrollo Sostenible.e mail-crodriguez@ minambiente.gov.co Fecha recepción: Mayo 19,2014 Fecha aprobación: Diciembre 16, $2014 \quad$ Editor asociado: Mantilla H.
} 
conducted, using the criteria of traffic lights, on the information obtained from 42 institutional planning documents and the answers to the requirement 2100-2916742009 of the Ministry of Environment, Housing and Territorial Development of Colombia (MAVDT), as well as some interviews with officials. Results: Except for one, all the CAR's submitted information on the implementation of this program in their jurisdictions (96.2\%). UAESPNN showed no physical or virtual media on this aspect, preventing their integral inclusion in the quantitative analysis. The action line with the highest percentage of direct implementation was the line I (in situ conservation: $30.8 \%$ ), the one with the highest indirect implementation percentage was action line $\mathrm{V}$ (institutional strengthening: $88.5 \%$ ), and the one with the highest non-implementation percentage was action line II (ex situ conservation: 42.3\%). Conclusions: The PNOA presents mostly an indirect implementation, where $61.5 \%$ of the evaluated CAR's only developed indirect activities related with it, associated with the implementation of other conservation programs. The evaluation exhibit an administrative gap inside the CAR's, showing that the actions implemented by them related with this program comes mostly from occasional actions than from institutional planning, reflecting that this program has not been yet structurally assimilated.

\section{Keywords: Andean bear, Environmental authorities,} National Conservation Program.

\section{Introducción}

El oso andino (Tremarctos ornatus) es la única especie de la familia Ursidae presente en Sudamérica. Actualmente es catalogado como vulnerable a la extinción (VU) por el Libro Rojo de Especies Amenazadas de la UICN y se encuentra listado en el Apéndice I de la Convención Internacional de Tráfico de Especies (CITES), según el cual está prohibida cualquier tipo de comercialización de individuos o sus partes. En Colombia es considerado como una especie en peligro de extinción bajo el criterio de vulnerable (Jorgenson et al. 2006). Su distribución está situada sobre la cordillera de los Andes, de donde toma su nombre, desde los 200 hasta los 4250 metros de elevación (Peyton 1980, Rodríguez et al. 1986, Castellanos 1996). Su hábitat incluye bosques húmedos, secos, matorral subdesértico y espinoso, desde las Sierras de Perijá colombo venezolana, de Mérida en Venezuela y el Darién colombo panameño en el norte, hasta Jujuy y Salta en Argentina, en el sur (Peyton 1980, Rodríguez et al. 2003, Del Moral y Bracho 2009). Aunque se ha mencionado la pre- sencia de la especie para la Sierra Nevada de Santa Marta (Solari et al.2013), actualmente no existe ninguna evidencia que soporte esta afirmación (ReyesAmaya 2015).

Aunque esta especie fue descrita desde el siglo XVIII, el trabajo orientado a su investigación y conservación se inició hace 24 años con las investigaciones de Bernard Peyton en Perú. Durante la última década el interés y la preocupación de un número creciente de personas y organizaciones por esta especie ha conducido a la creación de distintos frentes de trabajo que hoy desembocan en el desarrollo concertado de una estrategia para la implementación del Plan de Acción para la Conservación del Oso de Anteojos (Peyton 1999) y en la selección de acciones prioritarias por parte del Grupo de Especialistas en Osos para Sudamérica (ABET-UICN).

El Ministerio de Ambiente, Vivienda y Desarrollo Territorial (MAVDT) (2001) diseñó el Programa Nacional para la Conservación en Colombia del Oso Andino (Tremarctos ornatus) (PNOA), estableciendo las estrategias y acciones necesarias para garantizar su conservación, considerando la participación y compromiso de todos los actores involucrados. Dentro de las líneas de acción se definieron las siguientes:

Línea de Acción I: Conservación in situ. Mantener unidades de paisaje que contengan poblaciones de oso andino viables en el tiempo, a partir de la generación de información biológica y genética a nivel regional y local, con la ayuda de un sistema de información geográfica que asocie la anterior información, funcionando como herramienta para la toma de decisiones, unificando metodologías y procesos técnicos que permitan ejecutar proyectos comparables en las áreas de estudio e identificando las diferentes amenazas de tipo natural y antrópico para la priorización de determinadas áreas en el país para su conservación, mediante criterios biológicos, socioeconómicos y de oportunidades de conservación.

Línea de Acción II: Conservación ex situ. Adelantar estudios en cautiverio de osos andinos, relacionados con su biología, fisiología y ecología, identificando y caracterizando las condiciones de cautiverio adecuadas para desarrollar acciones de recuperación de individuos o poblaciones, así como la formulación de esquemas potenciales de recupera- 
ción y protección.

Línea de Acción III: Manejo sostenible. Definir e implementar un modelo de uso y manejo sostenible de las poblaciones naturales categorizadas como prioritarias para la conservación de la especie y establecer planes de manejo para la recuperación de estas, con base en su valoración y un esquema de compensación de daños, que favorezca una nueva interpretación de la especie por parte de las comunidades rurales.

Línea de Acción IV: Educación ambiental. Lograr que los actores involucrados en las relaciones hombre-oso alcancen actitudes y aptitudes positivas para la generación de un medio ambiente natural propicio para la conservación de la especie, tanto in situ como ex situ.

Línea de Acción V: Fortalecimiento institucional. Contar con personal capacitado y entrenado, apoyo científico, técnico administrativo y económico que permita el desarrollo y gestión en la escala nacional, regional y local de las diferentes acciones identificadas en el plan.

Por la necesidad de evaluar el estado de aplicación del PNOA, con el fin de establecer el impacto que este programa ha tenido al interior de las autoridades ambientales encargadas de su ejecisión, en el año 2010 se desarrolló en convenio con el MAVDT el proyecto de Evaluación del Programa Nacional para la Conservación en Colombia del Oso Andino (T. ornatus). De este proyecto se deriva el presente trabajo, partiendo de la inexistencia de documentos o bases de información sobre el presupuesto económico y las actividades específicas que se hubieran invertido y ejecutado a nivel nacional en las líneas de acción de este programa, desde el año de su creación hasta el año 2010. Se realizó una revisión, clasificación y análisis valorativo de la gestión de las CARs y de la UAESPNN, que son las autoridades ambientales por excelencia en sus áreas de jurisdicción según la Ley 99 de 1993 del Congreso de Colombia y el Decreto 3572 de 2011 del Departamento Administrativo de la Función Pública. Lo anterior, con el fin de establecer las debilidades y fortalezas en la planeación por parte de las instituciones evaluadas en la ejecución del programa, contribuyendo con información base para el establecimiento de prioridades en la conservación de esta especie en Colombia.

\section{Metodología}

Con el fin de evaluar la ejecución del PNOA se tomaron como insumo los diferentes programas de Gestión Ambiental Regional (PGAR), Planes de Acción Trienal (PAT), Planes de Ordenamiento y Manejo de Cuencas Hidrográficas (POMCA), informes de gestión y otros documentos de planeación (Tabla 1), que son emitidos por las autoridades ambientales que cuentan con la presencia de esta especie en su jurisdicción, según la distribución definida para esta especie en el país (Rodríguez et al. 2003) (Tabla 2). En los anteriores documentos se procedió a identificar los proyectos y actividades en relación con el PNOA que se hubieran implementado por estas entidades desde el 2001 año de creación del programa hasta el 2010. La obtención de esta información se efectuó mediante la revisión de las bibliotecas de las instituciones evaluadas, la realización de reuniones con funcionarios de las mismas y la revisión de las respuestas a la emisión del requerimiento 2100-2-91674 de 2009, donde el entonces llamado Ministerio de Ambiente, Vivienda y Desarrollo Territorial (MAVDT) solicitó a las CARs y a la UAESPNN, información sobre los proyectos, programas y actividades adelantados para la conservación de especies en peligro, entre las cuales se encuentra el oso andino. Debido al tipo de información con que se trabajó, esta evaluación abarcó la etapa de planeación, pero no el resultado o alcance de las acciones ejecutadas por las instituciones mencionadas.

En cada documento se realizó una revisión en busca de las acciones y actividades relacionadas con el PNOA, para realizar una evaluación cuantitativa en donde se identificaron las instituciones que hubieran o no aplicado este programa. La extracción de información se efectúo a dos niveles: en el primero se detectaron todas las actividades, programas y proyectos específicos relacionados directamente con el PNOA. En el segundo nivel se identificaron las actividades, programas y proyectos que aunque no nombran acciones relacionadas directamente con la especie, podrían implicar el cumplimiento del programa indirectamente, incidiendo en los ecosistemas asociados con la misma.

Para el análisis de la información obtenida se implementó un sistema semáforo, en el cual se eva- 
Tabla 1. Fuentes de información utilizadas para la evaluación cuantitativa del estado de aplicación del Programa Nacional para la Conservación en Colombia del Oso Andino (PNOA)*

\begin{tabular}{|c|c|}
\hline Documento o actividad & Descripción \\
\hline Planes de Gestión Ambiental Regional (PGAR) & $\begin{array}{l}\text { Instrumentos del Sistema Nacional Ambiental (SINA) para } \\
\text { dinamizar la gestión ambiental en las regiones, departamentos } \\
\text { y municipios. Estos planes son formulados con base en la } \\
\text { política ambiental nacional, para garantizar la articulación } \\
\text { funcional y programática de los ámbitos territorial y sectorial. }\end{array}$ \\
\hline $\begin{array}{l}\text { Planes de Acción y Planes de Acción Trienal } \\
\text { (PA y PAT) }\end{array}$ & $\begin{array}{l}\text { Herramienta para efectuar el seguimiento sectorial a la } \\
\text { planeación estratégica sectorial, a través del cumplimiento de } \\
\text { las metas establecidas en sus objetivos estratégicos. Este } \\
\text { seguimiento se realiza de manera trienal e incluye la gestión } \\
\text { adelantada por las entidades del sector y detalla las metas a las } \\
\text { que se comprometen en una vigencia determinada, fijando } \\
\text { tiempos y responsables. }\end{array}$ \\
\hline Informes de gestión & $\begin{array}{l}\text { Reflejan el desempeño de las actividades de gestión realizadas } \\
\text { en los ámbitos técnico, docente y administrativo. }\end{array}$ \\
\hline $\begin{array}{l}\text { Programa Nacional para la Conservación en } \\
\text { Colombia del Oso Andino (Tremarctos ornatus) }\end{array}$ & $\begin{array}{l}\text { Documento que establece las estrategias y las acciones que } \\
\text { garantizan la conservación de la especie, considerando la } \\
\text { participación y compromiso de todos los actores involucrados. }\end{array}$ \\
\hline $\begin{array}{l}\text { Requerimiento 2100-2-91674. Dirección de } \\
\text { Ecosistemas del MAVDT }\end{array}$ & $\begin{array}{l}\text { En este se solicitaba a las CARs y a algunas regionales de PNN } \\
\text { que informaran sobre los proyectos y programas adelantados } \\
\text { para la conservación de especies en peligro, entre las cuales se } \\
\text { encuentra el oso andino. }\end{array}$ \\
\hline $\begin{array}{l}\text { Reunión con funcionarios de la Unidad Adminis- } \\
\text { trativa Especial de Parques Nacionales Natura- } \\
\text { les (UAESPNN) }\end{array}$ & $\begin{array}{l}\text { Se abarcaron temas relacionados con el desarrollo de progra- } \\
\text { mas y/o proyectos implementados en las diferentes jurisdiccio- } \\
\text { nes de PNN. }\end{array}$ \\
\hline $\begin{array}{l}\text { Reunión con funcionario del Fondo de Compen- } \\
\text { sación Ambiental del MAVDT }\end{array}$ & $\begin{array}{l}\text { Se abarcaron temas sobre la financiación de programas } \\
\text { destinados a la conservación y protección del oso andino } \\
\text { mediante las CARs. }\end{array}$ \\
\hline
\end{tabular}

* Documentos de planeación, reuniones con funcionarios de las instituciones evaluadas y requerimiento 2100- 2-91674 de la Dirección de Ecosistemas del MAVDT a estas instituciones.

luó comparativamente qué autoridades ambientales invirtieron o ejecutaron presupuestos directa o indirectamente relacionados con cada una de las cinco líneas de acción del PNOA: en color rojo se clasificaron las CARs que no habían ejecutado proyectos o actividades directas o indirectas relacionadas con las diferentes líneas de acción del programa (no implementado); en color naranja se clasificaron las CARs que habían ejecutado proyectos o actividades que indirectamente podrían implicar la aplicación del programa (implementación indirecta); en color verde se clasificaron las CARs que habían ejecutado proyectos o actividades relacionadas directamente con las diferentes líneas de acción del programa (implementación directa).

A partir del año 2010 el conflicto oso-gente ha sido incluido como una sexta Línea de Acción del PNOA, sin embargo, en el presente documento lo concerniente a este aspecto ha sido incluido en la Línea de Acción III: Manejo Sostenible, porque durante el tiempo de ejecución del PNOA examinado en este trabajo se encontró incluido en este aparte.

\section{Resultados}

Con excepción de una, la totalidad de las CARs evaluadas presentaron información relacionada sobre si había o no desarrollado actividades directas o indirectas relacionadas con el PNOA (Tabla 3). La UAESPNN no suministró información física o digital que permitiera verificar o realizar un análisis cuantitativo de la ejecución del programa en sus regionales. Sin embargo se suministró información de forma verbal por parte de dos funcionarios de la UAESPNN, donde se menciona una actividad relacionada con el proceso de actualización del PNOA 
Tabla 2. Autoridades ambientales en las que fue evaluado el estado de aplicación del Programa Nacional para la Conservación en Colombia del Oso Andino (PNOA), en las cuales existe presencia de esta especie según el Programa Nacional para la Conservación en Colombia del Oso Andino (PNOA).

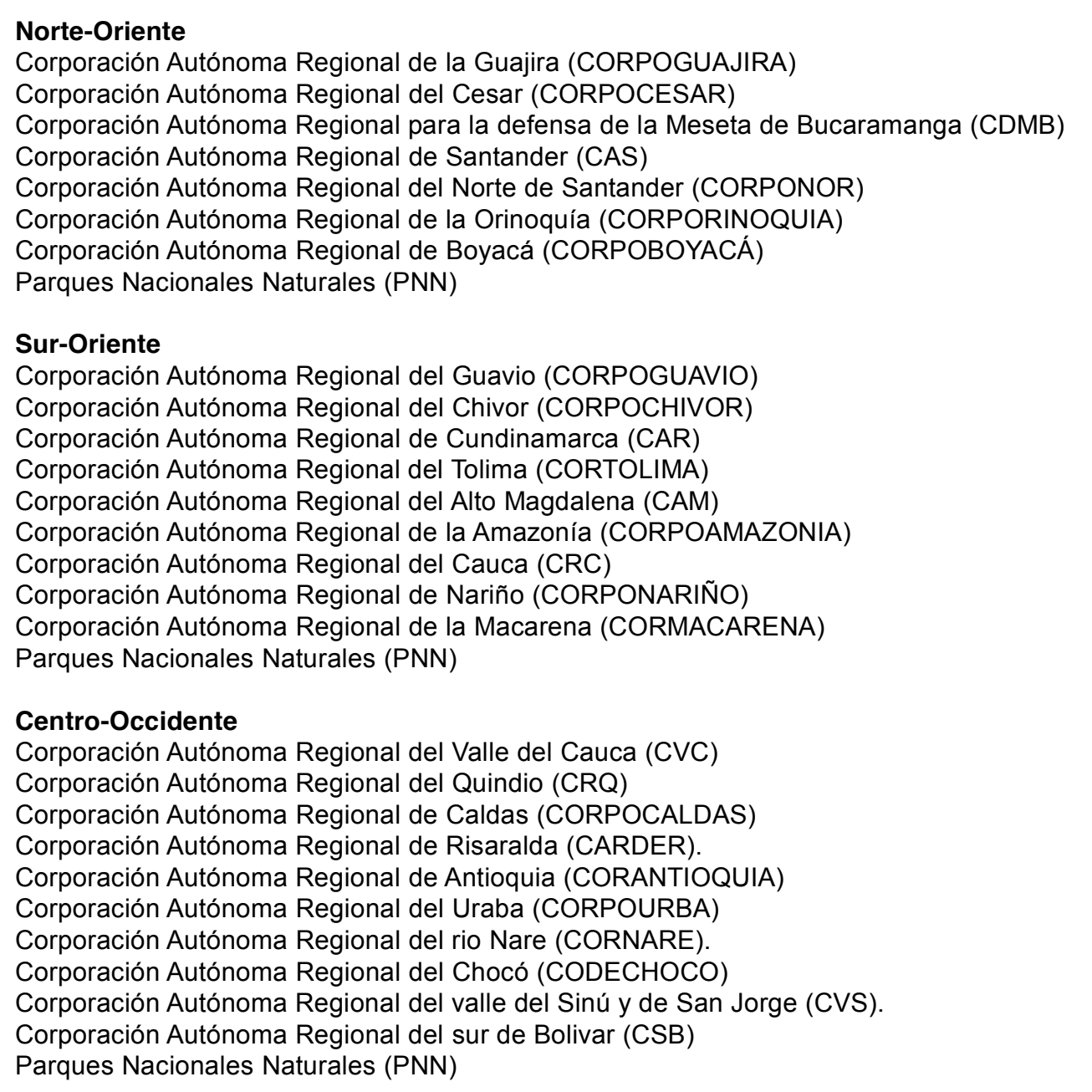

(2008), dos actividades de capacitación en metodologías para el monitoreo de la presencia de esta especie en su jurisdicción (2008 y 2010), así como del inicio de un proyecto piloto relacionado con este aspecto (2010) y una sobre capacitación en el manejo de los conflictos oso-gente (2009) (Tabla 4).

Línea de Acción I: Conservación in situ.Aunque se encontró que fueron ejecutados proyectos y acciones con cabida dentro de esta línea de acción por parte de la mayoría de las CARs, 65,4\% de los casos estuvieron orientados a la conservación del hábitat asociado, más con la especie, entre los que cuentan la declaración de nuevas áreas protegidas, así como esfuerzos por recuperar coberturas vegetales y la caracterización y zonificación ambiental de las ya existentes, incluyendo los Planes de Ordenamiento y Manejo de Cuencas Hidrográficas (POMCA) y otros planes de control y ordenamiento territorial. Un total de 30,8\% de las CARs desarrollaron actividades de monitoreo y zonificación de especies en vía de extinción involucrando específicamente al oso andino y la caracterización de los hábitats que este ocupa (Figura 1a).

Línea de Acción II: Conservación ex situ.Tan solo $19,2 \%$ de las CARs han adelantado acciones específicas sobre el oso andino en condiciones de cautiverio, tales como protocolos de manejo para individuos en proceso de rehabilitación y capacita- 
Tabla 3. Documentos de planeación suministrados por las corporaciones autónomas regionales (CARs) para la evaluación del estado de aplicación del Programa Nacional para la Conservación en Colombia del Oso Andino (PNOA).

\begin{tabular}{|c|c|c|c|}
\hline Corporación & $\begin{array}{l}\text { Activid } \\
\text { específicas }\end{array}$ & $\begin{array}{l}\text { lades } \\
\text { generales }\end{array}$ & Documentos presentados \\
\hline CORPOGUAJIRA & $x$ & $\mathrm{x}$ & PAT 2004-2007, PAT 2007-2011, PGAR \\
\hline CORPOCESAR & $\mathrm{x}$ & $\mathrm{x}$ & PA 2007-2011 \\
\hline CDMB & $\mathrm{x}$ & $\mathrm{x}$ & $\begin{array}{l}\text { Informe de gestión } 2004,2005,2006,2007,2008 \text { y } 2009 \text {. Plan } \\
\text { de acción ajustado 2007-2011. Plan anual de inversiones } 2010\end{array}$ \\
\hline CAS & $\mathrm{x}$ & $\mathrm{x}$ & PAT 2007-2011, PGAR \\
\hline CORPONOR & & $\mathrm{x}$ & PAT. 2004-2006, PAT. 2007-2009 \\
\hline CORPORINOQUIA & & $x$ & PAT 2007-2011, PGAR \\
\hline CORPOBOYACÁ & & $\mathrm{x}$ & PA 2007-2011 requerimiento 2100-2-91674 \\
\hline CORPOGUAVIO & $\mathrm{x}$ & $x$ & $\begin{array}{l}\text { PAT CORPOGUAVIO } 2007-2011 \text { requerimiento } 2100-2-91674 \text {, } \\
\text { Programa Nacional para la Conservación en Colombia del Oso } \\
\text { Andino (Tremarctos ornatus) }\end{array}$ \\
\hline CORPOCHIVOR & & $\mathrm{x}$ & PA 2007-2011 POAI 2010 \\
\hline CAR & $\mathrm{x}$ & $\mathrm{x}$ & $\begin{array}{l}\text { PAT 2004-2006. Informe de gestión } 2009 \text { (resumen ejecutivo). } \\
\text { PGAR 2001-2010. }\end{array}$ \\
\hline CORTOLIMA & & $\mathrm{x}$ & Requerimiento 2100-2-91674 \\
\hline CAM & & $\mathrm{x}$ & $\begin{array}{l}\text { PAT 2007-2009 PA 2007-2011 Programa nacional para la } \\
\text { conservación en Colombia del Oso Andino (Tremarctos } \\
\text { Ornatus) Requerimiento } 2100-2-91674\end{array}$ \\
\hline CORPOAMAZONIA & & $x$ & PA 2007-2011 requerimiento $2100-2-91674$ \\
\hline CRC & $\mathrm{x}$ & $\mathrm{x}$ & PA 2007-2011. POAI \\
\hline CORPONARIÑO & $x$ & $\mathrm{x}$ & PA 2007-2011. POAI requerimiento 2100-2-91674 \\
\hline CVC & & $\mathrm{x}$ & $\begin{array}{l}\text { PA 2007-2011. PAT 2007-2009. Doc. Metas y Presupuesto de } \\
\text { Gastos de Inversión Plan de Acción } 2010-2011 \text { requerimiento } \\
2100-2-91674\end{array}$ \\
\hline $\mathrm{CRQ}$ & & $x$ & PA 2007-2011. \\
\hline CARDER & & $x$ & PA 2007-2011. POAI requerimiento 2100-2-91674 \\
\hline CORANTIOQUIA & & $x$ & PA 2007-2011. POAI requerimiento 2100-2-91674 \\
\hline CORPOURBA & & $\mathrm{x}$ & PA 2007-2011 \\
\hline CORNARE & & & Requerimiento $2100-2-91674$ \\
\hline CODECHOCO & & $x$ & PA 2007-2011, PGAR \\
\hline CORPOCALDAS & & & Requerimiento 2100-2-91674 \\
\hline CSB & - & - & Esta Corporación no tiene documentos a disposición. \\
\hline CVS & & & Matriz de ajuste del PAT 2007-2011 \\
\hline CORMACARENA & & $\mathrm{x}$ & PA 2007-2012 requerimiento $2100-2-91674$ \\
\hline
\end{tabular}

ción a autoridades municipales sobre el manejo de los especímenes decomisados. El 38,5\% de las CARs evaluadas presentan proyectos y actividades relacionadas con la planeación sobre el manejo de especímenes en cautiverio, pero no es posible establecer si en estas se involucra al oso andino. El restante 42,3\% no presenta ningún proyecto o actividad relacionada con esta línea de acción (Figura 1b).

Línea de Acción III: Manejo sostenible. El $65,4 \%$ de las CARs presentaron proyectos o actividades en los documentos de gestión orientados con el desarrollo de sistemas productivos de desarrollo económico local o de investigación de las problemá- ticas, diseño de planes de control y realización de operativos contra el tráfico de fauna silvestre; sin embargo, no se encontró en esta información evidencia de una relación directa con el PNOA. Tan solo dos CARs $(7,7 \%)$ adelantaron actividades concretas con la especie relacionadas con la línea de manejo sostenible del PNOA. Con excepción del caso anterior, no se encontraron en los diferentes documentos de planeación actividades o proyectos que abordaran el fenómeno del conflicto oso-gente, asimismo en ningún caso se encontraron mediciones del impacto de los sistemas productivos sobre el hábitat o las poblaciones de oso andino. El restante 
Tabla 4. Información suministrada verbalmente por la Unidad Administrativa Especial de Parques Nacionales Naturales (UAESPNN).

\begin{tabular}{|c|c|c|}
\hline Responsables & Actividad & Año \\
\hline $\begin{array}{l}\text { Parques Nacionales Naturales (PNN) en } \\
\text { convenio con Wild Conservation Society (WCS) }\end{array}$ & Capacitaciones para miembros de las CAR y PNN & 2008 \\
\hline Parques Nacionales Naturales (PNN) & $\begin{array}{l}\text { Simposio Nacional del Programa Nacional para la } \\
\text { Conservación del Oso Andino en Colombia }\end{array}$ & 2008 \\
\hline $\begin{array}{l}\text { Parques Nacionales Naturales (PNN), Ministerio } \\
\text { de Ambiente, Vivienda y Desarrollo Territorial } \\
\text { (MAVDT) y World Society for the protection of } \\
\text { Animals (WSPA) }\end{array}$ & Taller sobre el Conflicto Oso-Hombre & 2009 \\
\hline $\begin{array}{l}\text { Parques Nacionales Naturales (PNN) en convenio } \\
\text { con Wild Conservation Society (WCS) }\end{array}$ & $\begin{array}{l}\text { Arranca el proyecto piloto de Monitoreo de la } \\
\text { especie al interior de PNN }\end{array}$ & 2010 \\
\hline $\begin{array}{l}\text { Parques Nacionales Naturales (PNN), Empresa } \\
\text { de Acueducto de Bogotá (EAB-ESP) y Corpora- } \\
\text { ción Autónoma Regional de Guavio } \\
\text { (CORPOGUAVIO) }\end{array}$ & $\begin{array}{l}\text { Taller para determinar las metodologías de } \\
\text { monitoreo de la especie }\end{array}$ & 2010 \\
\hline
\end{tabular}

26,9\% de las CARs no presentó proyectos o actividades relacionadas con esta línea de acción (Figura $1 \mathrm{c})$.

Línea de Acción IV: Educación Ambiental. Una gran parte de las corporaciones presentaron en sus planes de acción y gestión actividades de educación ambiental asociadas con la conservación y protección del medio ambiente y las especies de fauna amenazadas $(65,4 \%)$; sin embargo, no fue posible identificar su relación directa con la especie. En $15,4 \%$ de los casos fue posible encontrar proyectos y actividades educativas y de divulgación que involucraran específicamente a la especie y constituyeran un desarrollo directo del PNOA. El restante 19,2\% de las CARs no presentó avances en esta línea de acción (Figura 1d).

Línea de Acción V: Fortalecimiento institucional. Tan solo dos de las corporaciones $(7,7 \%)$ presentaron en los documentos revisados proyectos relacionados directamente con esta línea de acción, tales como convenios con otras instituciones en relación con la implementación del PNOA. Con excepción de una CAR que no presentó ningún tipo de actividad $(3,9 \%)$, las restantes presentaron actividades de fortalecimiento institucional que aunque no involucran al oso andino de forma directa, podrían incidir en la aplicación de esta línea de acción del PNOA $(88,5 \%)$ (Figura 1e).

\section{Discusión}

La Línea de Acción I: Conservación in situ, es la de mayor aplicación por parte de las autoridades ambientales de forma indirecta debido a la conjunción de otros programas nacionales y metas internacionales de conservación. Sin embargo, no es posible identificar si estas acciones indirectas están influyendo directamente de forma positiva sobre la especie, teniendo en cuenta que las actividades humanas son comunes a casi todas las áreas de distribución del oso andino y que su distribución en las zonas altas y medias de las montañas colombianas coinciden con el área de mayor desarrollo socioeconómico del país (Rodríguez y Cadena 1991, Peyton 1999), transformando los hábitats utilizados por el oso para su existencia.

La Línea de Acción II: Conservación ex situ, es la que presenta un mayor número de corporaciones que no han implementado actividades directas o indirectas en relación con ella; asimismo, en los casos en que se encontraron actividades posiblemente relacionadas con esta línea, debido a la naturaleza de la información colectada no fue posible identificar qué especies fueron cobijadas con estas actividades de planeación sobre el manejo de fauna silvestre en cautiverio. Si bien el PNOA no establece la conservación ex situ como una prioridad para la conservación de la especie en Colombia, resulta importante fortalecer esta línea de acción por el aumento de ejemplares extraídos de su hábitat y recuperados por las autoridades ambientales (Rodríguez et al.2005), no sólo en términos de su contención en cautiverio, sino en miras a la rehabilitación, liberación e incor- 

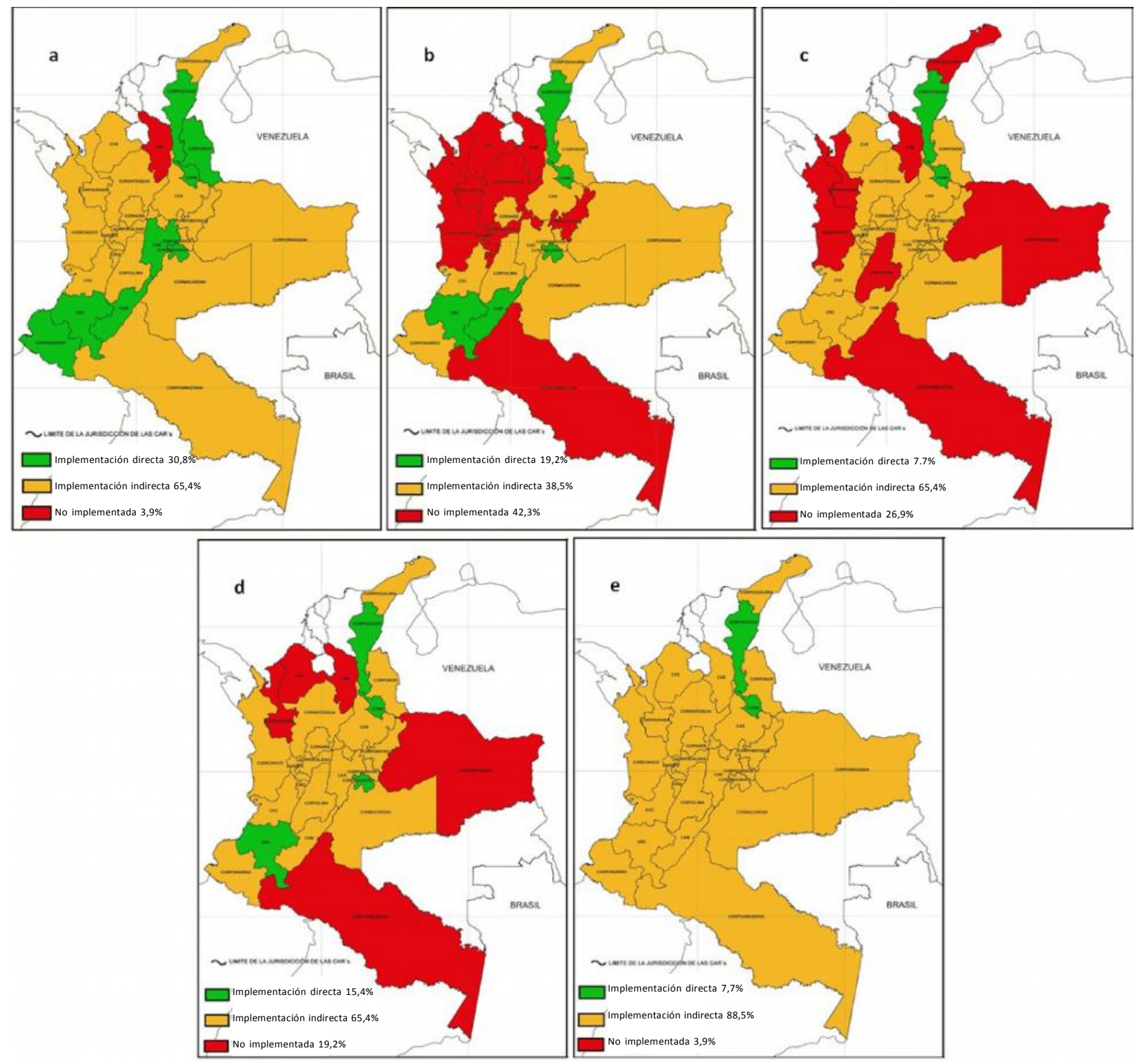

Figura 1. Estado de aplicación de las cinco líneas de acción del Programa Nacional para la Conservación en Colombia del Oso Andino (PNOA); a. Línea de Acción I (Conservación in situ), b. Línea de Acción II (Conservación ex situ), c. Línea de Acción III (Manejo sostenible), d. Línea de Acción IV (Educación ambiental), e. Línea de Acción V (Fortalecimiento institucional).

En color verde las corporaciones autónomas regionales (CARs) que planearon y ejecutaron proyectos o actividades relacionadas directamente con el PNOA (implementación directa). En color naranja las CARs que planearon y ejecutaron proyectos o actividades que indirectamente podrían implicar la aplicación del programa (implementación indirecta). En color rojo las CARs que no planearon o ejecutaron proyectos o actividades directas o indirectas relacionadas con el programa (no implementado).

poración efectiva de estos ejemplares a la vida silvestre.

En lo concerniente a la Línea de Acción III: Manejo Sostenible, se encontró que en esta línea las acciones desarrolladas por las instituciones tienen un impacto mayormente indirecto, donde los planes gestionados acerca del tráfico de especies han fortalecido indirectamente el cumplimiento de la misma, ayudando a desarrollar propuestas de incentivos y sanciones para la conservación de la especie.

Durante la evaluación realizada en esta investigación no existieron proyectos o programas conce- 
bidos dentro de la planeación de las distintas CARs examinadas para el manejo del conflicto oso-gente, lo cual demuestra que esta línea de acción aún requiere de un mayor esfuerzo institucional para su ejecución. Los conflictos entre los osos y la gente pueden causar pérdidas económicas y una excesiva tasa de mortalidad de osos (Poveda 1999, Jorgenson y Sandoval 2005), por lo que se hace necesaria la identificación y caracterización de esos conflictos en las áreas de presencia de la especie. De esta manera se podría conocer el grado de afectación tanto a los humanos como a los osos, con el fin de establecer programas, proyectos y actividades efectivas enfocadas a mitigar el conflicto, lo que permitiría disminuir considerablemente el peso económico de la conservación de los osos entre las comunidades humanas afectadas y mejorar el bienestar de ellos, creando un marco positivo para la coexistencia osogente.

La Línea de Acción IV: Educación Ambiental, presenta una gran limitante para evaluar su implementación, porque en la mayoría de los casos la información no muestra el tipo de campañas de educación ambiental realizadas, la comunidad objetivo y la relación de estas con el PNOA. Aunque se tienen proyectadas actividades de educación ambiental y campañas de divulgación, no existen indicadores de seguimiento, por lo que se desconoce el impacto positivo o negativo que han tenido en las comunidades a las que han sido dirigidas.

La Línea de Acción V: Fortalecimiento Institucional, por las limitaciones que la información analizada presenta no se puede llegar a evaluar qué tipo de convenios y trabajos han realizado las diferentes corporaciones con otras entidades, por lo que no se puede identificar con certeza el fortalecimiento institucional relacionado directamente con el PNOA. Asimismo resulta llamativo que esta línea de acción es la que presenta un menor grado de ejecución en relación directa con el PNOA. La importancia particular de esta línea de acción radica posiblemente en el uso que hacen de los hábitats las especies paisaje, que tienen altos requerimientos de área, heterogeneidad en el uso del hábitat, vulnerabilidad al uso humano de la tierra, funcionalidad ecológica y significado socioeconómico para las comunidades (Stern 1998, WCS 2002, Castellanos 2011). Estas especies paisaje en muchos casos se distribuyen bajo la jurisdicción de diferentes autoridades ambientales. Lo anterior hace que el fortalecimiento y la coordinación interinstitucional sean un aspecto clave para la conservación del oso andino, no sólo al interior de Colombia, sino recalcando la importancia de establecer programas de conservación binacionales con los países que se comparten fronteras con la presencia de esta especie, tal es el caso de la región norte de los Andes (Venezuela) y de la región sur (Ecuador).

Las áreas protegidas naturales constituyen un espacio relativamente efectivo para la conservación de la biodiversidad, sin embargo, tanto estas como las zonas de amortiguación se ven sometidas a una fuerte incidencia de las poblaciones humanas circundantes, las cuales ejercen efectos de extracción (cacería y extracción maderera), transformación (cultivos) e introducción (ganadería) (Rodríguez y Cadena 1991, Bruner et al.2001). Por esta razón, pese a que se tuvo conocimiento informal por parte de la UAESPNN sobre la existencia de algunas actividades dentro de los Parques Nacionales Naturales (PNN), relacionadas con el monitoreo de la presencia de la especie, el manejo del conflicto oso-gente, e incluso la actualización del PNOA, genera gran preocupación la ausencia de soportes de información física o virtual sobre la planeación y ejecución de programas, proyectos o actividades en relación con el programa.

En la mayoría de los documentos examinados en el presente trabajo no se encontraron los presupuestos invertidos para el desarrollo de las actividades específicas asociadas con el PNOA. A menudo estos fueron asignados de manera general, impidiendo determinar con estos datos el presupuesto asignado al PNOA.

Los programas y proyectos que afectan la aplicación del PNOA de manera indirecta hacen parte sobre todo de la implementación de lineamientos nacionales e internacionales. La Implementación del Sistema Regional de Áreas Protegidas (SIRAP) y del Sistema Nacional de Áreas Protegidas (SINAP) en Colombia y las Metas del Milenio acordadas por la Asamblea de Naciones Unidas en el 2000 se encuentran reflejadas en los planes de gestión. De igual manera se encuentran los programas y Proyectos Relacionados con el Manejo y Ordenamiento de Cuencas (POMCA). Algunos programas nacionales 
se reflejan de manera general en la gestión nacional y regional, afectando de manera indirecta a las poblaciones de osos. Entre ellos los más importantes por su peso en la gestión administrativa son el Programa para el Manejo Sostenible y Restauración de Ecosistemas de Alta Montaña en Colombia, la Estrategia Nacional para la Prevención y Control del Tráfico Ilegal de Especies Silvestres, y el Programa Nacional para la Conservación de Especies Amenazadas de Extinción.

A partir de la información obtenida en los Planes de Acción Trianual (PAT) no se puede hacer seguimiento eficaz a los proyectos planteados por las corporaciones, porque la mayoría de las veces estos quedan por fuera de los planes, o en estos no se muestra su avance, constituyendo un obstáculo para la evaluación y seguimiento del PNOA.

Tan solo 30,8\% de las CARs evaluadas presentaron actividades específicas relacionadas con alguna de las líneas de acción del PNOA (Tabla 3), de las cuales la mayoría se empezaron a desarrollar en el año 2009 o aún se encontraban en formulación para el año 2010. Esto revela la lenta incorporación del PNOA a los planes de gestión de las autoridades ambientales a nivel local, regional y nacional.

La información obtenida a través del requerimiento 2100-2-91674 de 2009 y las reuniones con funcionarios de las instituciones permitió conocer las actividades realizadas por estas, mientras que la revisión de los documentos de gestión permitió establecer las actividades planeadas con antelación por las mismas. De lo anterior fue posible concluir que existe una separación entre las actividades que se planean y aquellas que se realizan. La mayoría de las actividades desarrolladas por las corporaciones en relación con el PNOA no provienen de los documentos de planeación, por el contrario se desprenden de eventos coyunturales, como la radicación de denuncias sobre ataques de oso al ganado. Debido a lo anterior pueden existir actividades realizadas que no pudieron ser conocidas para la presente evaluación del PNOA.

\section{Conclusiones}

El Programa Nacional para la Conservación en Colombia del Oso Andino presenta una ejecución mayoritariamente indirecta, asociada más con la eje- cución de otros programas nacionales de conservación, de la cual es difícil inferir con certeza que se estén obteniendo logros concretos hacia la conservación de esta especie en Colombia.

Existe un vacío administrativo al interior de las autoridades ambientales con responsabilidad de ejecutar el PNOA, en donde las acciones ejecutadas por las mismas en relación con este provienen de acciones coyunturales y no de la planeación institucional. Esto muestra que el PNOA no ha sido asimilado aun de forma estructural por las CARs, lo que dificulta el correcto funcionamiento de estas en relación con la conservación de esta especie en Colombia.

La revisión bibliográfica de los planes de acción y gestión de las CARs no constituye por sí misma una herramienta completa para la evaluación del PNOA, porque es limitada la calidad y cantidad de información que se puede obtener de estos documentos. La fuente de información más precisa sobre las actividades y proyectos involucrados directa o indirectamente con el PNOA durante el presente trabajo fue el Requerimiento Na 2100-2-91674 de la Dirección de Ecosistemas del MAVDT y no los documentos de gestión.

\section{Agradecimientos}

Al Ministerio de Ambiente y Desarrollo Sostenible y a las 26 corporaciones autónomas incluidas en el análisis, por facilitar la información necesaria para el desarrollo de este estudio.

\section{Literatura citada}

Bruner A, Gullison R, Rice R, Da Fonseca G. 2001. Effectiveness of parks in protecting tropical biodiversity. Science. 291 (5): $125-8$.

Castellanos A. 1996. Liberación de tres osos de anteojos en la Reserva Biológica Maquipucuna, Ecuador. Informe final. Programa Libearty/Ecuador, World Society for the Protection of Animals. 21 p.

Castellanos A. 2011. Andean bear home ranges in the Intag region, Ecuador. Ursus. 22 (1) : 65-73.

Congreso de Colombia. 1993. Ley 99, Por la cual se crea el Ministerio del Medio Ambiente, se reordena el sector público encargado de la gestión y conservación del medio ambiente y los recursos naturales renovables, se organiza el Sistema Nacional Ambiental (SINA) y se dictan otras disposiciones. Bogotá: Congreso de Colombia; $44 \mathrm{p}$.

Del Moral F, Bracho E. 2009. Indicios indirectos de la presencia del oso andino (Tremarctos ornatus, Cuvier, 1825) en el 
noroeste de Argentina. Rev Mus Argent Cienc Natur. 11 (1): 69-76.

Departamento Administrativo de la Función Pública. 2011. Decreto 3572, Por el cual se crea una Unidad Administrativa Especial, se determinan sus objetivos, estructura y funciones. Bogotá: Departamento Administrativo de la Función Pública; 12 p.

Jorgenson JP, Patiño M, Silva L. 2006. Oso de anteojos Tremarctos ornatus. En: Rodríguez-Mahecha JV, Alberico M, Trujillo F, Jorgenson J (eds). Libro Rojo de los Mamíferos de Colombia. Bogotá: Conservación Internacional Colombia, Ministerio de Ambiente, Vivienda y Desarrollo Territorial. p. 381-6.

Jorgenson JP, Sandoval S. 2005. Andean bear management needs and interactions with humans in Colombia. Ursus 16 (1): 108-16.

Ministerio de Ambiente, Vivienda y Desarrollo Territorial. 2010. Resolución 383, Por la cual se declaran las especies silvestres que se encuentran amenazadas en el territorio nacional y se toman otras determinaciones. Bogotá: Ministerio de Ambiente, Vivienda y Desarrollo Territorial; $29 \mathrm{p}$.

Ministerio del Medio Ambiente. 2001. Programa Nacional para la Conservación en Colombia del Oso Andino (Tremarctos ornatus). Bogotá: Ministerio del Medio Ambiente; 27 p.

Peyton B. 1980. Ecology, distribution, and food habits of spectacled bears, Tremarctos ornatus in Perú.J Mammal. 61 (4): 639-52.

Peyton B. 1999. Spectacled bear conservation action plan. En: Servheen CS, Herrero S, Peyton B (eds). Bears, status survey and conservation action plan. Gland: IUCN/SSC Bear and polar bear specialist group. p. 157-98.

Poveda J. 1999. Interacciones ganado-oso en límites de 5 municipios con el Parque Natural Chingaza: una aproximación cartográfica. (Trabajo de grado). Bogotá: Facultad de Ciencias, Pontificia Universidad Javeriana; $94 \mathrm{p}$.

Reyes-Amaya N. 2015. Familia Ursidae. En: Suárez-Castro AF, Ramírez-Cháves HE. (eds.). Los carnívoros terrestres y semiacuáticos continentales de Colombia. Bogotá: Editorial Universidad Nacional de Colombia. In press

Rodríguez D, Cadena A. 1991. Evaluación y calidad del hábitat del oso andino Tremarctos ornatus en el Parque Nacional Natural Las Orquídeas y zonas adyacentes, Antioquia, Colombia. (Trabajo de grado). Bogotá: Facultad de Ciencias, Universidad Nacional de Colombia. 24 p.

Rodríguez D, Cuesta F, Goldstein I, Naranjo L, Hernández O. 2003. Estrategia ecorregional para la conservación del oso andino Tremarctos ornatus en los Andes del norte. Bogotá: WWF, Fundación Wii, Ecociencia, Wildlife Conservation Society; $38 \mathrm{p}$.

Rodríguez D, Feliciano O, Rodríguez C. Análisis histórico sobre el origen y destino final de los oseznos capturados en Colombia. Bogotá: Fundación para la Investigación, Conservación y Protección del Oso Andino (Wii), Fundación Biocolombia, Ministerio de Ambiente, Vivienda y Desarrollo Territorial. (En línea) 2005 (Acceso 12 de diciembre). URL https://www.sugarsync.com/pf/ D7976082_4677294_66662

Rodríguez D, Poveda F, Rivera D, Sánchez J, Jaimes V, Lozada R. 1986. Reconocimiento preliminar del hábitat natural del oso andino (Tremarctos ornatus) y su interacción con el hombre en la región nororiental del Parque Nacional Natural El Cocuy. Bol Div Manaba. 1 (1): 1-47.

Solari S, Muñoz-Saba Y, Rodríguez-Mahecha JV, Defler TR, Ramírez-Chaves HE, Trujillo F. 2013. Riqueza, endemismo y conservación de los mamíferos de Colombia. Mastozool Neotrop. 20: 301-65.

Stern SJ. 1998. Field studies of large mobile organisms: scale, movement and habitat utilization. En: Peterson DL, Parker VT (eds). Ecological scale: theory and applications. New York: Columbia University Press. p. 289-307.

Wildlife Conservation Society. Selecting landscape species.. (En línea) 2002 (Acceso 10 de diciembre) URL http:// www.wcslivingland scapes.org/DesktopModules/ Bring 2mind/DMX/Download .aspx \%3FEntryId\%3 D5 184\%26PortalId\%3D0\%26DownloadMethod $\% 3$ Dattachment+\&cd=1\&hl=es\&ct=clnk\&gl=co 